\title{
Cascading Gravity: Extending the Dvali-Gabadadze-Porrati Model to Higher Dimension
}

\author{
Claudia de Rham, ${ }^{1,2}$ Gia Dvali, ${ }^{3,4}$ Stefan Hofmann, ${ }^{1,5}$ Justin Khoury, ${ }^{1}$ Oriol Pujolàs, ${ }^{4}$ \\ Michele Redi, ${ }^{4,6}$ and Andrew J. Tolley ${ }^{1}$ \\ ${ }^{1}$ Perimeter Institute for Theoretical Physics, 31 Caroline St. N., Waterloo, ON, N2L 2Y5, Canada \\ ${ }^{2}$ Department of Physics \& Astronomy, McMaster University, Hamilton ON, L8S 4M1, Canada \\ ${ }^{3}$ CERN, Theory Division, CH-1211 Geneva 23, Switzerland \\ ${ }^{4}$ Center for Cosmology and Particle Physics, New York University, New York, New York 10003, USA \\ ${ }^{5}$ NORDITA, Roslagstullsbacken 23, 10691 Stockholm, Sweden \\ ${ }^{6}$ Institut de Théorie des Phénomènes Physiques, EPFL, CH-1015, Lausanne, Switzerland
}

(Received 15 January 2008; published 27 June 2008)

\begin{abstract}
We present a generalization of the Dvali-Gabadadze-Porrati scenario to higher codimensions which, unlike previous attempts, is free of ghost instabilities. The 4D propagator is made regular by embedding our visible 3-brane within a 4-brane, each with their own induced gravity terms, in a flat $6 \mathrm{D}$ bulk. The model is ghost-free if the tension on the 3-brane is larger than a certain critical value, while the induced metric remains flat. The gravitational force law "cascades" from a 6D behavior at the largest distances followed by a $5 \mathrm{D}$ and finally a $4 \mathrm{D}$ regime at the shortest scales.
\end{abstract}

DOI: 10.1103/PhysRevLett.100.251603

PACS numbers: 11.25.Wx, 04.50.Kd, 98.80.Cq

Introduction. - The present acceleration of the Universe is a profound mystery. While the observational data are consistent with a cosmological constant $(\mathrm{CC})$ of order $\left(10^{-3} \mathrm{eV}\right)^{4}$, this value is in stark disagreement with particle physics computations. The problem is even more severe than the hierarchy problem in the Standard Model, since dynamical solutions are impossible in theories with a massless $4 \mathrm{D}$ graviton [1]. In the same way as the perihelion precession of Mercury was explained by a modification of Newtonian gravity, an alternative approach is to assume that the acceleration signals a breakdown of general relativity at cosmological distances.

The Dvali-Gabadadze-Porrati (DGP) model [2] provides a simple mechanism to modify gravity at large distances by adding a localized graviton kinetic term on a codimension 1 brane in a flat 5D spacetime. The extension to higher dimensions is particularly important both for its possible embedding into string theory and for its relevance to the $\mathrm{CC}$ problem $[3,4]$. However, the natural generalization of the DGP model with higher codimension branes is not straightforward $[5,6]$. On the one hand, these models require some regularization due to the divergent behavior of the Green's functions in higher codimension. More seriously, most constructions are plagued by ghost instabilities around flat space (not to be confused with those of the selfaccelerating branch of standard 5D DGP) [5,6] - see [7] for related work. The purpose of this Letter is to show that both pathologies can be resolved by embedding the codimension 2 DGP model into a codimension 1 brane with its own kinetic term. It will be interesting to see if this setup allows for higher-codimension self-accelerated solutions. Our present focus, however, is to derive a consistent framework in which gravity is modified in the infrared.

Scalar. - We shall focus on the codimension 2 case. As a warm-up, we consider a real scalar field with action,

$$
S=\frac{1}{2} \int \phi\left[M_{6}^{4} \square_{6}+M_{5}^{3} \square_{5} \delta(z)+M_{4}^{2} \square_{4} \delta(y) \delta(z)\right] \phi
$$

describing a codimension 2 kinetic term embedded into a codimension 1 one in 6D. We will impose throughout the Letter $Z_{2} \times Z_{2}$ orbifold projection identifying $y \rightarrow-y$ and $z \rightarrow-z$. The model possesses the two mass scales, $m_{5}=$ $M_{5}^{3} / M_{4}^{2}$ and $m_{6}=M_{6}^{4} / M_{5}^{3}$.

In absence of the $4 \mathrm{D}$ kinetic term, the propagator on the codimension 1 brane (4-brane) is the DGP propagator [2],

$$
G^{0}\left(y-y^{\prime}\right)=\frac{1}{M_{5}^{3}} \int_{-\infty}^{\infty} \frac{d q}{2 \pi} \frac{e^{i q\left(y-y^{\prime}\right)}}{p^{2}+q^{2}+2 m_{6} \sqrt{p^{2}+q^{2}}},
$$

where $y$ is the coordinate orthogonal to the codimension 2 brane (3-brane), $p$ the 4D momentum, and $q$ the momentum along $y$. To find the exact 5D propagator, we can treat the $4 \mathrm{D}$ kinetic term (located at $y=0$ ) as a perturbation and then sum the series. One finds,

$$
\begin{aligned}
G\left(y, y^{\prime}\right)= & G^{0}\left(y-y^{\prime}\right)-M_{4}^{2} G^{0}(y) p^{2} G^{0}\left(-y^{\prime}\right) \\
& +M_{4}^{4} G^{0}(y) p^{4} G^{0}(0) G^{0}\left(-y^{\prime}\right)+\ldots \\
= & G^{0}\left(y-y^{\prime}\right)-\frac{M_{4}^{2} p^{2}}{1+M_{4}^{2} p^{2} G^{0}(0)} G^{0}(y) G^{0}\left(-y^{\prime}\right) .
\end{aligned}
$$

In particular, the $4 \mathrm{D}$ brane-to-brane propagator is determined in terms of the higher dimensional Green's function, $G_{4}=G(0,0)=G^{0}(0) /\left[M_{4}^{2} G^{0}(0) p^{2}+1\right]$.

For the case at hand,

$$
G^{0}(0)=\frac{2}{\pi M_{5}^{3} \sqrt{4 m_{6}^{2}-p^{2}}} \tanh ^{-1}\left(\sqrt{\frac{2 m_{6}-p}{2 m_{6}+p}}\right) .
$$

For $p>2 m_{6}$, the analytic continuation of this expression is understood. 
Remarkably, the 5D kinetic term makes the 4D propagator finite, thereby regularizing the logarithmic divergence characteristic of pure codimension 2 branes. In particular, when $M_{5}$ goes to zero, one has $G_{4} \rightarrow$ $M_{6}^{-4} \log \left(p / m_{6}\right)$, reproducing the codimension 2 Green's function with a physical cutoff given by $m_{6}$. The corresponding 4D Newtonian potential scales as $1 / r^{3}$ at the largest distances, showing that the theory becomes six dimensional, and reduces to the usual $1 / r$ on the shortest scales. Its behavior at intermediate distances, however, depends on $m_{5,6}$. If $m_{5}>m_{6}$, there is an intermediate 5D regime; otherwise, the potential directly turns $6 \mathrm{D}$ at a distance of order $\left(m_{5} m_{6}\right)^{-1 / 2} \log \left(m_{5} / m_{6}\right)$.

Gravity.-Let us now turn to gravity. In analogy with the scalar field, we consider the action,

$$
S=\frac{M_{6}^{4}}{2} \int \sqrt{-g_{6}} R_{6}+\frac{M_{5}^{3}}{2} \int \sqrt{-g_{5}} R_{5}+\frac{M_{4}^{2}}{2} \int \sqrt{-g_{4}} R_{4}
$$

where each term represents the Ricci scalar. This guarantees that the model is fully $6 \mathrm{D}$ general covariant.

To find the propagator, it is convenient to follow the same procedure as for the scalar and sum the diagrams with insertion of the lower dimensional kinetic term, i.e., the Einstein tensor $\mathcal{E}$. For our purpose, we only compute the propagator on the 3-brane. Given the higher dimensional propagator, the brane-to-brane propagator due to the insertion of a codimension 1 term is, in compact form,

$$
G_{\mu \nu \alpha \beta}=\left\{G^{0}\left[1-M_{4}^{2} \mathcal{E} G^{0}\right]^{-1}\right\}_{\mu \nu \alpha \beta}=G_{\mu \nu \gamma \delta}^{0} H_{\alpha \beta}^{\gamma \delta},
$$

where the first equality refers to the same resummation as in (2) and $G_{\mu \nu \gamma \delta}^{0}$ is the $4 \mathrm{D}$ part of the higher dimensional Green's function evaluated at zero. The tensor $H_{\alpha \beta}^{\mu \nu}$ satisfies by definition,

$$
\left[1-M_{4}^{2} \mathcal{E} G^{0}\right]_{\gamma \delta}^{\mu \nu} H_{\alpha \beta}^{\gamma \delta}=\frac{1}{2}\left(\delta_{\mu}^{\alpha} \delta_{\nu}^{\beta}+\delta_{\mu}^{\beta} \delta_{\nu}^{\alpha}\right) .
$$

To find $H$, one can write the most general Lorentz covariant structure compatible with the symmetries,

$$
\begin{aligned}
H_{\alpha \beta}^{\gamma \delta}= & a\left(\delta_{\alpha}^{\gamma} \delta_{\beta}^{\delta}+\delta_{\alpha}^{\delta} \delta_{\beta}^{\gamma}\right)+b \eta^{\gamma \delta} \eta_{\alpha \beta}+c\left(p^{\gamma} p_{\alpha} \delta_{\beta}^{\delta}\right. \\
& \left.+p^{\delta} p_{\alpha} \delta_{\beta}^{\gamma}+p^{\gamma} p_{\beta} \delta_{\alpha}^{\delta}+p^{\delta} p_{\beta} \delta_{\alpha}^{\gamma}\right)+d p^{\gamma} p^{\delta} \eta_{\alpha \beta} \\
& +e \eta^{\gamma \delta} p_{\alpha} p_{\beta}+f p^{\gamma} p^{\delta} p_{\alpha} p_{\beta},
\end{aligned}
$$

where $\eta_{\alpha \beta}$ represents the flat Minkowski metric. Requiring that this satisfies Eq. (5) leads to a system of linear equations whose solution determines the coefficients $a, b, c, d, e, f$. Using this information, one then reconstructs the exact propagator from Eq. (4).

It is straightforward to apply this technique to cascading DGP. Starting from 6D, the propagator on the 4-brane is [4]

$$
G_{M N P Q}=\frac{\tilde{\eta}_{M P} \tilde{\eta}_{N Q}+\tilde{\eta}_{M Q} \tilde{\eta}_{N P}-\frac{1}{2} \tilde{\eta}_{M N} \tilde{\eta}_{P Q}}{2 M_{5}^{3}\left(p_{5}^{2}+2 m_{6} p_{5}\right)}
$$

where $\tilde{\eta}_{M N}=\eta_{M N}+\frac{p_{M} p_{N}}{2 m_{6} p_{5}}$, and $M, N \ldots$ are $5 \mathrm{D}$ indices and $p_{5}^{2}=p_{M} p^{M} . G_{\mu \nu \alpha \beta}^{0}$ is obtained by integrating the 5D propagator with respect to the extra momentum. To compute the propagator on the 3-brane, we determine the coefficients $a, b, c, d, e, f$ through the system of linear equations (5). One finds,

$$
\begin{aligned}
& a=c / I_{1}=-\frac{1}{2\left(I_{1} p^{2}+1\right)} \\
& b=-p^{2} d=\frac{I_{1} p^{2}}{\left(I_{1} p^{2}+1\right)\left(I_{1} p^{2}-2\right)} \\
& e=\frac{1}{3\left(I_{1} p^{2}+1\right)}-\frac{4 I_{1}+3 I_{2} p^{2}}{3\left(I_{1} p^{2}-2\right)} \\
& f=\frac{I_{2}+2 I_{1}^{2}+I_{1} I_{2} p^{2}}{\left(I_{1} p^{2}+1\right)\left(I_{1} p^{2}-2\right)}
\end{aligned}
$$

where $I_{1}=M_{4}^{2} G^{0}(0) / 2$ with $G^{0}(0)$ defined in (3) and

$$
\begin{aligned}
I_{2}= & \frac{1}{2 m_{6} m_{5}} \int_{-\infty}^{\infty} \frac{d q}{2 \pi} \\
& \times \frac{1}{\sqrt{p^{2}+q^{2}}\left(p^{2}+q^{2}+2 m_{6} \sqrt{p^{2}+q^{2}}\right)} .
\end{aligned}
$$

All these coefficients are finite, showing that the regularization is also effective for the spin 2 case.

Having determined the coefficients of the tensor $H$, the full propagator is given by Eq. (4). To linear order the amplitude between two conserved sources on the brane is rather simple,

$$
-\frac{1}{M_{4}^{2}} \frac{I_{1}}{I_{1} p^{2}+1}\left(T_{\mu \nu} T^{\prime \mu \nu}-\frac{I_{1} p^{2}-1}{2 I_{1} p^{2}-4} T T^{\prime}\right),
$$

and only depends on the first integral $I_{1}$.

The coefficient in front of the amplitude is exactly as for the scalar; however, there is a nontrivial tensor structure. One worrisome feature of this amplitude is that the relative coefficient of $T_{\mu \nu} T^{\prime \mu \nu}$ and $T T^{\prime}$ interpolates between $-1 / 4$ in the IR and $-1 / 2$ in the UV. The $-1 / 4$ in the IR gives the correct tensor structure of gravity in 6D and is unavoidable because at large distances, the physics is dominated by $6 \mathrm{D}$ Einstein term. From the 4D point of view, this can be understood as the exchange of massive gravitons and an extra-scalar. The $-1 / 2$ in the UV, on the other hand, signals the presence of a ghost. This agrees with previous results $[5,6]$ which used a different regularization. From the 4D point of view, the theory decomposes into massive spin 2 fields and scalars. Since the massive spin 2 gives an amplitude with relative coefficient $-1 / 3$, the extra repulsion must be provided by a scalar with wrong sign kinetic term. Separating from Eq. (8) the massive spin two contribution, we identify the scalar propagator as

$$
G_{\text {ghost }}=\frac{1}{6 M_{4}^{2}} \frac{I_{1}}{I_{1} p^{2}-2} .
$$

This propagator has a pole with a negative residue; there- 
fore, it contains a localized (tachyonic) ghost mode in addition to a continuum of healthy modes.

Ghost free theory. - To clarify the origin of the ghost, it is illuminating to consider the decoupling limit studied in [8]. This will allow us to show how a healthy theory can be obtained by simply introducing tension on the 4D brane while retaining a flat intrinsic geometry.

In the $6 \mathrm{D}$ case, the decoupling limit [8] corresponds to taking $M_{5}, \quad M_{6} \rightarrow \infty$ with $\Lambda_{s} \equiv\left(m_{6}^{2} M_{5}^{3 / 2}\right)^{2 / 7}=$ $\left(M_{6}^{16} / M_{5}^{9}\right)^{1 / 7}$ finite. In this limit, the physics on the 4-brane admits a local 5D description, where only the nonlinearities in the helicity 0 part of the metric are kept, and are suppressed by the scale $\Lambda_{s}$. The effective 5D Lagrangian is given by

$$
\begin{aligned}
L_{5}= & \frac{M_{5}^{3}}{8} h^{M N}(\mathcal{E} h)_{M N}-\frac{3}{2} M_{5}^{3}(\partial \pi)^{2}\left(1+\frac{9}{32 m_{6}^{2}} \square_{5} \pi\right) \\
& +\delta(z)\left(\frac{M_{4}^{2}}{8} \tilde{h}^{\mu \nu}(\mathcal{E} \tilde{h})_{\mu \nu}+\frac{1}{2} \tilde{h}^{\mu \nu} T_{\mu \nu}\right),
\end{aligned}
$$

where $(\mathcal{E} h)_{M N}=\square h_{M N}+\ldots$ is the linearized Einstein tensor, $M, N \ldots$ are 5D indices, and $\mu, \nu \ldots$ are four dimensional. We have rescaled $\pi$ and $h_{\mu \nu}$ so that they are dimensionless, and the physical 5D metric perturbation is

$$
\tilde{h}_{M N}=h_{M N}+\pi \eta_{M N} .
$$

The first line of (10) is the 5D version of the " $\pi$ Lagrangian" introduced in [8] for the DGP model. In addition to this, we have the localized curvature term on the 3-brane, which depends on 4D physical metric $\tilde{h}_{\mu \nu}$. This introduces a kinetic mixing between $\pi$ and the 5D metric.

We now take a further step and compute the boundary effective action valid on the 3-brane. At the quadratic order, by integrating out the fifth dimension, the 5D kinetic term of $\pi$ produces a $4 \mathrm{D}$ "mass term" $\sim M_{5}^{3} \sqrt{-\square_{4}}$ while the Einstein tensor gives rise to Pauli-Fierz (PF) structure for $h_{\mu \nu}$ on the boundary [9],

$$
\begin{aligned}
L_{4}= & -\frac{M_{5}^{3}}{4} h^{\mu \nu} \sqrt{-\square_{4}}\left(h_{\mu \nu}-h \eta_{\mu \nu}\right)-3 M_{5}^{3} \pi \sqrt{-\square_{4}} \pi \\
& +\frac{M_{4}^{2}}{8} \tilde{h}^{\mu \nu}(\mathcal{E} \tilde{h})_{\mu \nu}+\frac{1}{2} \tilde{h}^{\mu \nu} T_{\mu \nu}
\end{aligned}
$$

where $h_{\mu \nu}$ and $\pi$ now denote the 5D fields evaluated at the 3-brane location.

In terms of the physical metric, (12) takes the form,

$$
\begin{aligned}
L_{4}= & -\frac{M_{5}^{3}}{4} \tilde{h}^{\mu \nu} \sqrt{-\square_{4}}\left(\tilde{h}_{\mu \nu}-\tilde{h} \eta_{\mu \nu}\right)-\frac{3}{2} M_{5}^{3} \pi \sqrt{-\square_{4}} \tilde{h} \\
& +\frac{M_{4}^{2}}{8} \tilde{h}^{\mu \nu}(\mathcal{E} \tilde{h})_{\mu \nu}+\frac{1}{2} \tilde{h}^{\mu \nu} T_{\mu \nu} .
\end{aligned}
$$

Note that the kinetic term for $\pi$ is completely absorbed by that of $\tilde{h}_{\mu \nu}$, and only a cross term between $\pi$ and $\tilde{h}_{\mu}^{\mu}$ remains.

From this, it is straightforward to show the presence of a ghost. The scalar longitudinal component of $h_{\mu \nu}$ acquires a positive kinetic term by mixing with the graviton $[8,10]$. By taking

$$
\tilde{h}_{\mu \nu}=\hat{h}_{\mu \nu}+\phi \eta_{\mu \nu}+\frac{\partial_{\mu} \partial_{\nu}}{m_{5} \sqrt{-\square_{4}}} \phi,
$$

one finds that there are in fact two 4D scalar modes whose kinetic matrix in the UV is

$$
\frac{3}{4} M_{4}^{2} \square_{4}\left(\begin{array}{ll}
1 & 1 \\
1 & 0
\end{array}\right)
$$

which has obviously a negative eigenvalue corresponding to a ghost.

Having understood the origin of the ghost, we are now ready to show how to cure it. To achieve this, we clearly need to introduce a positive localized kinetic term for $\pi$. This can arise from extrinsic curvature contributions. The simplest and most natural choice is to put a tension $\Lambda$ on the 3-brane. This produces extrinsic curvature while leaving the metric on the brane flat since the tension only creates a deficit angle.

The solution to the 5D equations following from (10) for a 3-brane with tension $\Lambda$ is [11]

$$
\pi^{(0)}=\frac{\Lambda}{6 M_{5}^{3}}|z|, \quad h_{\mu \nu}^{(0)}=-\frac{\Lambda}{6 M_{5}^{3}}|z| \eta_{\mu \nu} .
$$

This is an exact solution including the nonlinear terms for $\pi$ - they vanish identically for this profile. The background corresponds to a locally flat $6 \mathrm{D}$ bulk with deficit angle $\Lambda / M_{6}^{4}$ and with flat $4 \mathrm{D}$ sections.

The crucial point is that on this background, the $\pi$ Lagrangian acquires contributions from the nonlinear terms. These can be found considering the perturbations,

$$
\begin{aligned}
& \pi=\pi^{(0)}(z)+\delta \pi\left(z, x^{\mu}\right), \\
& h_{\mu \nu}=h_{\mu \nu}^{(0)}(z)+\delta h_{\mu \nu}\left(z, x^{\mu}\right), \\
& T_{\mu \nu}=-\Lambda \eta_{\mu \nu}+\delta T_{\mu \nu} .
\end{aligned}
$$

Plugging (17) in (10) and dropping $\delta$, one obtains at quadratic order (up to a total derivative),

$$
\delta L_{5}=-\frac{9}{8} \frac{\Lambda}{m_{6}^{2}} \delta(z)\left(\partial_{\mu} \pi\right)^{2} .
$$

This is a localized kinetic term for $\pi$ that contributes to the 4D effective action with a healthy sign when $\Lambda>0$. Therefore, for large enough $\Lambda$, the kinetic matrix for the 2 scalars (15) becomes positive, and the ghost is absent.

This can also be seen by computing the one particle exchange amplitude. With the addition of (18), the effective $4 \mathrm{D}$ equations are 


$$
M_{4}^{2}(\mathcal{E} \tilde{h})_{\mu \nu}-2 M_{5}^{3} \sqrt{-\square_{4}}\left(\tilde{h}_{\mu \nu}-\tilde{h} \eta_{\mu \nu}\right)=-2 T_{\mu \nu}+6 M_{5}^{3} \sqrt{-\square_{4}} \pi \eta_{\mu \nu}
$$

$$
\frac{3 \Lambda}{2 m_{6}^{2}} \square_{4} \pi=M_{5}^{3} \sqrt{-\square_{4}} \tilde{h} .
$$

Using the Bianchi identities and the conservation of $T_{\mu \nu}$, the double divergence of (19) leads to

$$
M_{5}^{3} \sqrt{-\square_{4}}\left[(\mathcal{E} \tilde{h})_{\mu}^{\mu}+6 \square_{4} \pi\right]=0,
$$

where we have used $(\mathcal{E} \tilde{h})_{\mu}^{\mu}=2\left(\partial^{\mu} \partial^{\nu} \tilde{h}_{\mu \nu}-\square_{4} \tilde{h}\right)$. On the other hand, the trace of (19), in conjunction with (20) and (21), leads to

$$
M_{4}^{2} \mathcal{O}_{(\pi)} \pi=-2 T_{\mu}^{\mu},
$$

where $\mathcal{O}_{(\pi)} \equiv\left[9\left(\Lambda / m_{6}^{2} M_{4}^{2}\right)-6\right] \square_{4}-24 m_{5} \sqrt{-\square_{4}}$.

Combining (19), (21), and (22), one derives that the physical metric is, up to pure gauge terms,

$$
\tilde{h}_{\mu \nu}=\frac{-2}{M_{4}^{2}}\left\{\frac{1}{\mathcal{O}}\left(T_{\mu \nu}-\frac{T}{3} \eta_{\mu \nu}\right)+\frac{1}{\mathcal{O}_{(\pi)}} T \eta_{\mu \nu}\right\},
$$

where $\mathcal{O}=\square_{4}-2 m_{5} \sqrt{-\square_{4}}$. The tensor structure of the amplitude interpolates between $-1 / 4$ in the IR and

$$
-\frac{1}{3}+\frac{1}{6}\left(\frac{3}{2} \frac{\Lambda}{M_{4}^{2} m_{6}^{2}}-1\right)^{-1}
$$

in the UV. The amplitude above corresponds to the exchange of massive spin 2 fields and a scalar obeying Eq. (22). The (DGP-like) kinetic term for the scalar is positive as long as

$$
\Lambda>\frac{2}{3} M_{4}^{2} m_{6}^{2} .
$$

In this regime, we see that the localized ghost disappears and the scalar sector is composed of a healthy resonance.

In the limit we are considering, the tension required is consistent with having six noncompact dimensions. Indeed, requiring that the deficit angle in the bulk be less than $2 \pi$ leads to $\Lambda<2 \pi M_{6}^{4}$. It follows that

$$
3 \pi M_{5}^{6}>M_{4}^{2} M_{6}^{4},
$$

which is always satisfied in the $5 D$ decoupling limit and displays the necessity of the induced term on the codimension 1 brane. Moreover, the condition above is equivalent to having $m_{6}<m_{5}$, which suggests that in order to avoid the ghost, one should cascade from the highest dimension down to 4D "step by step."

From a phenomenological point of view, observations require that $m_{5} \lesssim H_{0}$, the present Hubble scale. The most interesting possibility is when the $6 D$ crossover scale is larger but of similar order. Assuming that the formulas above can be extrapolated in this regime for a Planckian $M_{4}$, this implies that $M_{5}$ is of order $10 \mathrm{MeV}$ and $M_{6} \sim$ $\mathrm{meV}$. The latter also sets the scale of $\Lambda$.

Discussion. - In this Letter, we have presented a six dimensional DGP model with cascading localized kinetic terms. The model interpolates between a $6 \mathrm{D}$ behavior at large distances and a 4D one at short distances with an intermediate $5 \mathrm{D}$ regime. The kinetic terms regularize the divergent codimension 2 behavior. The model is ghost-free at least for a certain range of parameters if on the codimension 2 brane there is a large enough tension.

We have left several questions for future study. At linearlevel, the tensor structure of the graviton propagator is inconsistent with observations. In the context of DGP, this was shown not to be a problem because the nonlinearities restore the correct tensor structure [12]. A hint of a similar phenomenon in the present model is given by the longitudinal terms of the graviton propagator (4). These are singular when the mass parameters $m_{5,6}$ vanish and give large contributions to nonlinear diagrams. In fact, we expect a "double" Vainshtein effect. For dense enough sources, the nonlinearities should first decouple the extra 5D scalar mode restoring 5D behavior followed by another step to 4D. Another important direction to study is cosmology. The model has the intriguing codimension 2 feature that tension does not curve the space. This is obviously of interest for the cosmological constant problem.

We thank Gregory Gabadadze for useful discussions. This work is supported in part by NSF Grant No. PHY0245068 and by the David and Lucile Packard Foundation (G. D. and M. R.), by DURSI under Grant No. 2005 BP-A 10131 (O.P.), and by NSERC and MRI (J. K., A. J.T., C.d.R., and S.H.).

[1] S. Weinberg, Rev. Mod. Phys. 61, 1 (1989).

[2] G. R. Dvali, G. Gabadadze, and M. Porrati, Phys. Lett. B 484, 112 (2000).

[3] G. Dvali, G. Gabadadze, and M. Shifman, Phys. Rev. D 67, 044020 (2003).

[4] G. R. Dvali and G. Gabadadze, Phys. Rev. D 63, 065007 (2001).

[5] S. L. Dubovsky and V.A. Rubakov, Phys. Rev. D 67, 104014 (2003).

[6] G. Gabadadze and M. Shifman, Phys. Rev. D 69, 124032 (2004).

[7] N. Kaloper and D. Kiley, J. High Energy Phys. 05 (2007) 045 .

[8] M. A. Luty, M. Porrati, and R. Rattazzi, J. High Energy Phys. 09 (2003) 029; A. Nicolis and R. Rattazzi, J. High Energy Phys. 06 (2004) 059.

[9] The massless 5D graviton decomposes in a continuum of massive 4D gravitons. Therefore, in the unitary gauge, the boundary effective action will have PF structure.

[10] N. Arkani-Hamed, H. Georgi, and M. D. Schwartz, Ann. Phys. (N.Y.) 305, 96 (2003).

[11] G. Dvali, G. Gabadadze, O. Pujolas, and R. Rahman, Phys. Rev. D 75, 124013 (2007).

[12] C. Deffayet, G. Dvali, G. Gabadadze, and A. Vainshtein, Phys. Rev. D 65, 044026 (2002). 